\title{
Using Operando Electrochemical TEM as Part of a Correlative Approach to Characterize Failure Modes in Solid-state Energy Storage Devices
}

Nikhilendra Singh ${ }^{1}$, James Horwath ${ }^{2}$, Timothy Arthur ${ }^{1}$, Daan Hein Alsem ${ }^{3}$ and Eric Stach ${ }^{2}$

${ }^{1}$ Toyota Research Institute of North America, Ann Arbor, Michigan, United States, ${ }^{2}$ University of Pennsylvania, Philadelphia, Pennsylvania, United States, ${ }^{3}$ Hummingbird Scientific, Lacey, Washington, United States

Looking at next generation batteries one can distinguish two directions: further engineering optimizations in current lithium-ion battery technology and fundamental studies on new emerging energy storage systems like all-solid state, multivalent or Li-air batteries. Solid state batteries can provide competitive energy densities and may also provide potential safety advantages over traditional Li-ion batteries. Because solid electrolytes have a higher shear modulus, they should suppress Li dendrite growth, a common cause of shorting in Li-ion cells. However, in practice solid electrolytes display decomposition upon contact with $\mathrm{Li}$ and the growth of $\mathrm{Li}$ dendrites through the solid electrolyte, still causing potential shorts. Ideally one would design solid-state batteries with an ideal Solid Electrolyte Interface (SEI) to suppress or eliminate such failure modes.

It has been shown that using additives in bulk solid electrolyte (SE) materials like $\mathrm{Li}_{3} \mathrm{PS}_{4}(\mathrm{LPS})$ results in a robust SEI at the Li metal/SE interface [1]. Here we study failure modes in LPS electrolyte materials with and without the addition of a halide. With purpose-designed operando transmission electron microscope (TEM) biasing sample holders, it is possible to make very localized electrical contacts to a sample inside the TEM using a movable probe and study the Li-metal and solid-electrolyte surfaces insitu to gain additional insight into what causes the identified failure modes (Figure 1). This method has previously been used to investigate, the switching of oxide resistive memories [2], anode materials for batteries $[3,4]$, and the conductance through rows of atoms $[5,6]$.

Using the in-situ biasing sample holder and Energy Dispersive X-ray Spectroscopy in the TEM, we confirmed that simply bringing the surface of the lithium metal in contact with LPS:0.5Lil caused the iodine to diffuse through the lithium metal (i.e. through the Li/LPS:0.5Lil interface) as shown in Figure 2. Furthermore, we used the tool to perform in-situ electrochemical cycling. Micron-sized particles were contacted with a mobile probe and cyclic voltammograms were obtained. Currents in the order of picoamps were measured and, simultaneously, electrochemically induced structural changes, within particles and at interfaces, were imaged in the TEM. Comparing LPS and LPS:0.5Lil show significant differences in how Li metal plates and strips from both electrolytes. Using in-situ TEM biasing sample holder tools we were able to directly image such structural changes.
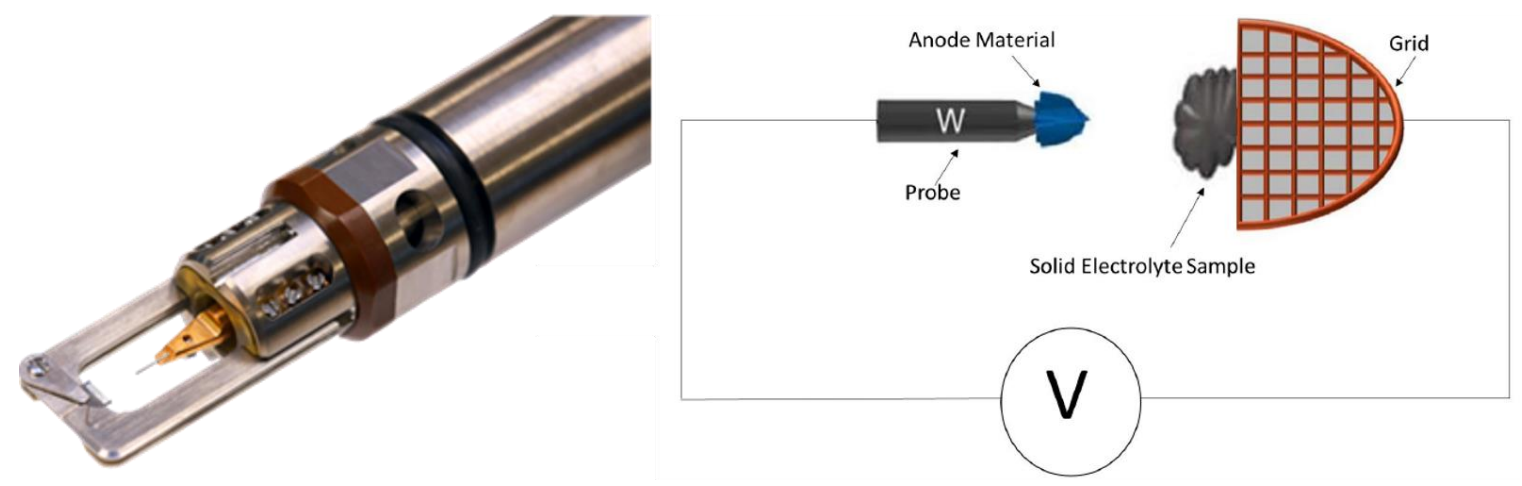
Figure 1. From left to right, tip of the operando TEM biasing sample holder and detailed schematic of the experiment.

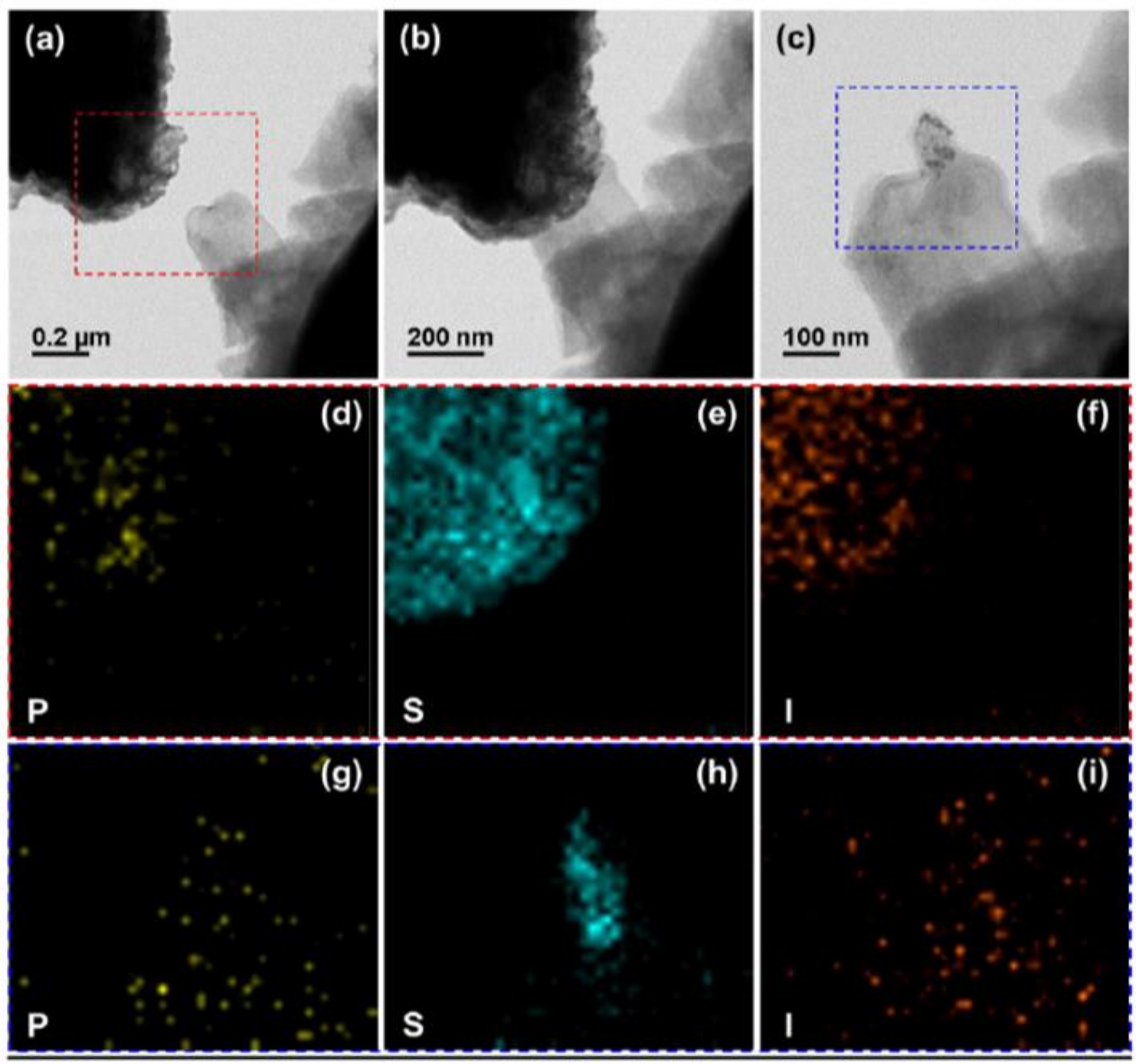

Figure 2. In-situ TEM shows that iodine before contact (a, d, e and f) is contained within the "bulk" of the LPS:0.5Lil, but upon contact (b) and after contact (c) diffuses through the Li metal (g, h, i).

\section{References}

[1] Han et.al., Adv. Energy Mater., 8 (18), 1703644 (2018).

[2] David Cooper, et al., Advanced Materials 29 (2017), p. 1700212.

[3] Fei-Hu, et al., ACS Nano 11 (2017), p. 8628.

[4] Ze Yang, et al., Energy Storage Materials 9 (2017), p. 214.

[5] Hideaki Ohnishi, Yukihito Kondo, Kunio Takayanagi. Nature 395 (1998), p. 780. 